\section{Bapen symposium: "original communications" OC-032 OUTCOME ON HOME PARENTERAL NUTRITION: 33 YEARS EXPERIENCE FROM A NATIONAL CENTRE}

doi:10.1136/gutjnl-2012-302514a.32

${ }^{1} \mathrm{M}$ Dibb, ${ }^{*} \mathrm{G}$ Carlson, ${ }^{3} \mathrm{~A}$ Bradley, ${ }^{2}$ Anderson, ${ }^{3} \mathrm{~J}$ Shaffer, ${ }^{3} \mathrm{~A}$ Teubner, ${ }^{3} \mathrm{~S}$ Lal. ${ }^{1}$ Department of Gastroenterology, Salford Royal Foundation NHS Trust, Salford, UK; ${ }^{2}$ Department of Surgery, Salford Royal Foundation NHS Trust, Salford, UK; ${ }^{3}$ Intestinal Failure Unit, Salford Royal Foundation NHS Trust, Salford, UK

Introduction Home parenteral nutrition (HPN) is an essential treatment modality for patients with Type 3 intestinal failure (IF), but long term data on the factors associated with HPN dependence and survival are limited.

Methods Medical records of patients with IF who received HPN for more than 3 months from a national IF Unit between 1978 and 2011 were reviewed. Kaplan-Meier curves and Cox regression analysis were performed to identify factors associated with HPN dependence and poor prognosis.

Results Case notes of 547 patients were reviewed. The overall probability of survival was $89 \%, 67 \%, 58 \%$ and $27 \%$ at $1,5,10$ and 20 years after starting treatment. Multivariate analysis demonstrated an association between diagnosis and survival; Crohn's disease, mesenteric ischaemia and pseudo-obstruction were associated with a better outcome. There was also an association with increasing age at IF diagnosis and poor outcome. There was no association between small bowel length or the occurrence of catheter-related infection and survival. 14.7\% (25/170) of deaths were related to HPN complications. Line sepsis and IF-associated liver disease were considered to be the cause of death in 10 and 15 patients respectively (mean survival of these patients: 67 months (range 6-223); 32\% within the first 2 years of treatment).

Continued HPN dependence in survivors was $83 \%, 63 \%, 59 \%$ and $53 \%$ at 1, 5, 10 and 15 years respectively. $80 \%$ of those who achieved nutritional independence from HPN did so within the first 7 years of treatment. Surgical reconstruction resulted in a shorter period of HPN dependence than adaptation and/or medical therapies (19.8 vs 32.8 months respectively) $p=0.008$. On multivariate analysis, mechanism of IF was significantly associated with the likelihood of HPN independence: patients with gastro-intestinal dysmotility or malabsorption were less likely to achieve HPN independence, whereas those with short bowel syndrome with or without enterocutaneous fistula were more likely achieve HPN independence. Of the latter group, patients with a small bowel length $>150 \mathrm{~cm}$ were $3.5 \times$ more likely to achieve nutritional independence compared to those with $<50 \mathrm{~cm}$.

Conclusion This is the largest reported long-term experience of survival and dependence on HPN and will inform future decisions about evolving alternative therapies for type 3 IF, such as small bowel transplantation and lengthening. Nutritional autonomy can be achieved in a significant proportion of patients through adaptation, medical treatment of underlying disease and/or surgical reconstruction.

Competing interests None declared.

\section{OC-033 \\ TAUROLIDINE SIGNIFICANTLY REDUCES THE INCIDENCE OF CATHETER RELATED BLOOD STREAM INFECTIONS IN PATIENTS ON HOME PARENTERAL NUTRITION}

doi:10.1136/gutjnl-2012-302514a.33

${ }^{1} \mathrm{~J}$ A Saunders, ${ }^{*}{ }^{1} \mathrm{M}$ Naghibi, ${ }^{1} \mathrm{~T}$ R Smith, ${ }^{2} \mathrm{~A}$ T King, ${ }^{3} \mathrm{Z}$ B Leach, ${ }^{1} \mathrm{M}$ A Stroud. ${ }^{1} \mathrm{NIHR}$ Nutrition, Diet and Lifestyle BRU, Southampton, UK; ${ }^{2}$ Department of Surgery, University Hospital Southampton, Southampton, UK; ${ }^{3}$ Department of Nutrition and Dietetics, University Hospital Southampton, Southampton, UK

Introduction Catheter related blood stream infections (CRBSI) in patients receiving home parenteral nutrition (HPN) cause significant morbidity, are potentially life-threatening and through repeated line replacements may compromise venous access. We introduced products containing taurolidine as preventative antimicrobial line locks in 2006 for HPN patients who had repeated episodes of CRBSI and report the incidence of CRBSI before and after

Methods Data were retrospectively collected from electronic and paper records for all adult HPN patients between January 2001 and October 2011.

Results Data were available for 19/22 HPN patients receiving taurolidine locks for a period of 51.9 patient years (21.5 years pre-taurolidine and 30.4 years post-taurolidine). Patients were offered taurolidine if they had: (1) two or more episodes of community borne CRBSI (2) one episode of CRBSI in patients with a persistent source of intra-abdominal sepsis (3) significantly compromised vascular access. The use of taurolidine significantly reduced the rate of CRBSI from 4.59 to 0.81 episodes per 1000 days $(p<0.0001)$. Nine episodes of recurrent CRBSI occurred in five patients despite taurolidine (one patient had four further infections in 5.6 years). The organisms responsible were coag neg staph. (four episodes), pseudomonas (one episode), candida (one episode), mixed coliform and coag neg staph (one episode) and two cases which were culture negative.

Conclusion Our experience shows that taurolidine significantly reduces the rate of CRBSI, particularly in patients who have had at least two previous infections. It may also have a role in preventing infection in patients with foci of potential abdominal sepsis in the context of type 2 IF. Despite this, it should not be seen as a substitute for meticulous line access techniques as some patients continue to develop recurrent infections while on treatment.

Competing interests None declared.

\section{OC-034 SALVAGE OF CENTRAL VENOUS CATHETERS IN HPN CATHETER-RELATED BLOOD STREAM INFECTIONS IS SAFE AND EFFECTIVE: 18 YEARS EXPERIENCE FROM A NATIONAL CENTRE}

doi:10.1136/gutjnl-2012-302514a.34

${ }^{1} \mathrm{M}$ Dibb, ${ }^{*}{ }^{2} \mathrm{G}$ Carlson, ${ }^{2} \mathrm{~A}$ Abraham, ${ }^{3} \mathrm{~J}$ Shaffer, ${ }^{3} \mathrm{~A}$ Teubner, ${ }^{3} \mathrm{~S}$ Lal. ${ }^{1}$ Department of Gastroenterology, Salford Royal Foundation NHS Trust, Salford, UK; ${ }^{2}$ Department of

Abstract OC-033 Table 1

\begin{tabular}{|c|c|c|c|c|c|c|c|}
\hline & $\mathbf{n}$ & Male & $\begin{array}{l}\text { Median age at } \\
\text { HPN initiation } \\
\text { years }\end{array}$ & $\begin{array}{l}\text { Duration HPN } \\
\text { treatment prior } \\
\text { to taurolidine } \\
\text { (median) days }\end{array}$ & $\begin{array}{l}\text { Episodes of CRBSI } \\
\text { per } 1000 \text { patient } \\
\text { days pre-taurolidine } \\
\text { (number of episodes) }\end{array}$ & $\begin{array}{l}\text { Duration of HPN } \\
\text { treatment } \\
\text { post-taurolidine } \\
\text { (median) days }\end{array}$ & $\begin{array}{l}\text { Episodes of CRBSI } \\
\text { per } 1000 \text { patient days } \\
\text { post-taurolidine } \\
\text { (number of episodes) }\end{array}$ \\
\hline All patients & 19 & 10 & 55 & $7846(162)$ & $4.59(36)$ & $11088(371)$ & $0.81 *(9)$ \\
\hline 2 or more episodes CRBSI & 11 & 7 & 39 & $5188(256)$ & $6.17(32)$ & $6505(348)$ & $1.23^{*}(8)$ \\
\hline 1 CRBSI plus persistent source of abdominal sepsis & 4 & 1 & 71 & $24(0)$ & $0(0)$ & $845(226)$ & $0(0)$ \\
\hline Compromised vascular access & 4 & 2 & 59 & $2634(207)$ & $1.51(4)$ & $3738(812)$ & $0.27 \dagger(1)$ \\
\hline
\end{tabular}

${ }^{*} p$ Value $<0.0001$ (proportion testing)

tp Value 0.08 (proportion testing). 